\title{
Dynamical aspects of supercooled TIP3P- water in the grooves of DNA
}

Cite as: J. Chem. Phys. 150, 235101 (2019); https://doi.org/10.1063/1.5100601

Submitted: 18 April 2019 . Accepted: 31 May 2019 . Published Online: 19 June 2019

(D) M. A. F. dos Santos, (D) M. A. Habitzreuter, M. H. Schwade, R. Borrasca, M. Antonacci, G. K. Gonzatti, (D) P. A. Netz, and (iD) M. C. Barbosa

\section{COLLECTIONS}

Paper published as part of the special topic on Chemical Physics of Supercooled Water SCH2O2O19
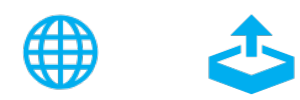

\section{ARTICLES YOU MAY BE INTERESTED IN}

Water dynamics in the hydration shells of biological and non-biological polymers

The Journal of Chemical Physics 150, 234904 (2019); https://doi.org/10.1063/1.5096392

Is water one liquid or two?

The Journal of Chemical Physics 150, 234503 (2019); https://doi.org/10.1063/1.5096460

Diffusion dynamics of supercooled water modeled with the cage-jump motion and hydrogen-bond rearrangement

The Journal of Chemical Physics 150, 204502 (2019); https://doi.org/10.1063/1.5095978

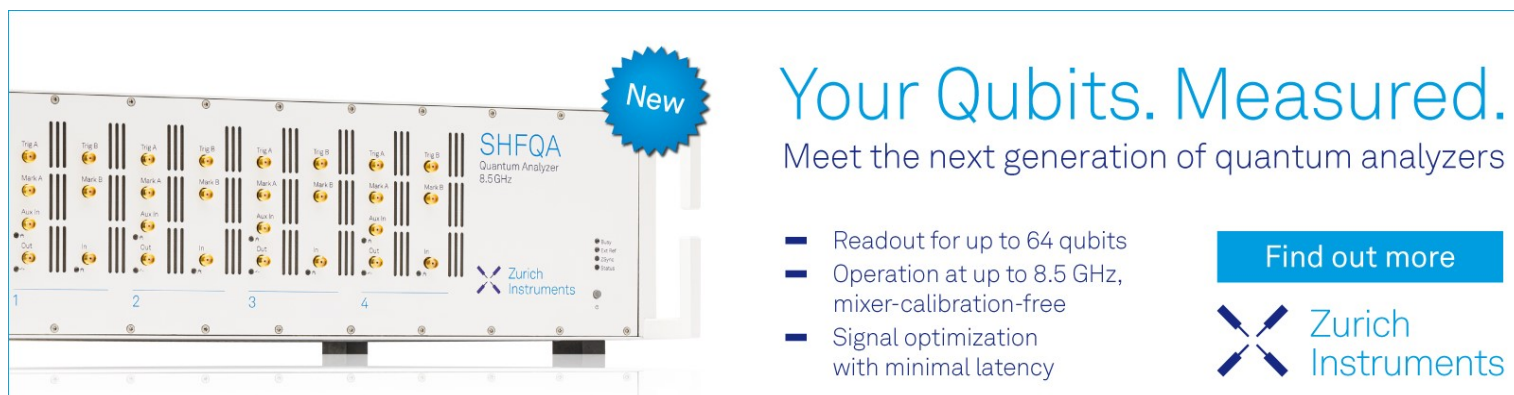




\title{
Dynamical aspects of supercooled TIP3P-water in the grooves of DNA
}

\author{
Cite as: J. Chem. Phys. 150, 235101 (2019); doi: 10.1063/1.5100601 \\ Submitted: 18 April 2019 • Accepted: 31 May 2019 • \\ Published Online: 19 June 2019
}

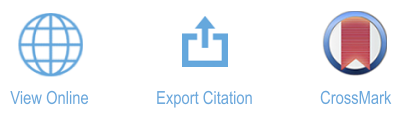

\author{
M. A. F. dos Santos, ${ }^{1}$ (D) M. A. Habitzreuter, ${ }^{1}$ (D) M. H. Schwade, ${ }^{1}$ R. Borrasca, ${ }^{1}$ M. Antonacci, \\ G. K. Gonzatti, ${ }^{2}$ P. A. Netz, ${ }^{2}$ (D) and M. C. Barbosa ${ }^{3, a)}$ (D)
}

\begin{abstract}
AFFILIATIONS
${ }^{1}$ Instituto de Física, Universidade Federal do Rio Grande do Sul, Caixa Postal 15051, CEP 91501-970, Porto Alegre, RS, Brazil

${ }^{2}$ Instituto de Química, Universidade Federal do Rio Grande do Sul, CEP 91501-970, Porto Alegre, RS, Brazil

${ }^{3}$ Instituto de Física, Departamento de Física, Universidade Federal do Rio Grande do Sul, Caixa Postal 15051,

CEP 91501-970, Porto Alegre, RS, Brazil
\end{abstract}

Note: This paper is part of a JCP Special Topic on Chemical Physics of Supercooled Water.

author to whom correspondence should be addressed: marcia.barbosa@ufrgs.br

\begin{abstract}
We investigate by molecular dynamics simulations the mobility of the water located at the DNA minor and major grooves. We employ the TIP3P water model, and our system is analyzed for a range of temperatures 190-300 K. For high temperatures, the water at the grooves shows an Arrhenius behavior similar to that observed in the bulk water. At lower temperatures, a departure from the bulk behavior is observed. This slowing down in the dynamics is compared with the dynamics of the hydrogen of the DNA at the grooves and with the autocorrelation functions of the water hydrogen bonds. Our results indicate that the hydrogen bonds of the water at the minor grooves are highly correlated, which suggests that this is the mechanism for the slow dynamics at this high confinement.
\end{abstract}

Published under license by AIP Publishing. https://doi.org/10.1063/1.5100601

\section{INTRODUCTION}

Water is ubiquitous in nature. Notwithstanding the simplicity of the molecule, water presents more than 70 anomalies in the thermodynamic, dynamic, and structural behavior. ${ }^{1}$ One example is the unusual high mobility of water at low temperatures which increases under pressure. ${ }^{2}$ This mobility becomes even more unusual in nanoconfined environments ${ }^{3-7}$ where water violates the macroscopic hydrodynamic equations by exhibiting superflow and by not obeying the Stokes Einstein relation. ${ }^{8}$ This discovery turns nanoconfined systems into potential filters for desalination processes. $^{9-11}$

Water also plays a key role in the dynamics of bioactive materials such as polymers, proteins, and cells. ${ }^{12-19}$ The presence of water at the surface of proteins enables these molecules to display several conformations in addition to the lowest energy configurations. The possibility of having these conformations allows the protein to perform activities that are fundamental for the existence of life. One example of the relevance of water in biosystems is that dry proteins are unable to perform these functionalities.
In addition to the effects water causes in other materials, water properties are also affected by the presence of the macromolecules. Water in the vicinity of hydrophobic and hydrophilic sites of the protein exhibits a dynamical behavior which is quite distinct when compared to bulk water. ${ }^{20-27}$ Recently, experiments and simulations with water at the GFP and CYP proteins showed that water molecules at the biomolecule surface present a very slow dynamics and they get stuck in some places, suggesting the presence of fractal traps on the protein surface. ${ }^{28,29}$ In these systems, the water near the protein exhibits a subdiffusive behavior in which the mean squared displacement (MSD) of the molecules grows nonlinearly with time, i.e., $\left\langle r^{2}(t)\right\rangle \propto t^{\mu}$, in which $\mu<1$ for subdiffusion. ${ }^{3}$

Even though the slow dynamics at the protein surface is experimentally observed, the quantitative evaluation of the subdiffusive behavior is not trivial. The computational investigation of the diffusive aspects of water in the hydration layer of biomolecules presents major challenges since during the diffusion, the molecules may leave the region that defines the layer of hydration, making it difficult to track water molecules at the contact layer. In addition, the structure of the proteins displays a huge diversity, what makes it 
difficult to look for universality. This means that the mechanisms which cause the peculiar diffusive behavior of hydration water might depend on the specific nature of the protein and on the specific region in the protein surface. In some cases, the slow dynamics is due to the spatial limitations of the confining geometry, with the surface acting as a fractal structure; ${ }^{28}$ in other cases, the slow mobility in the hydration water occurs due to the hydrogen bonds whose strength and lifetime depends on the hydrophilicity of the surface. $^{26,27,31}$

The scientific literature has reported dynamical transitions on hydration water of two types: a shift to higher temperatures of the dynamic transition already expected for bulk water and new dynamic crossovers due to the water-biomolecule interaction. One example of the first case is the experimental results of Chen et al. ${ }^{32}$ which showed a water fragile-to-strong dynamic crossover similar to the transition expected for bulk water when the molecules are confined to a biomolecule surface. One example of the second type of transition was found by Gallo et al. ${ }^{25}$ when they analyzed the relation between slow dynamics of hydration-water and the dynamical transition of proteins. In particular, the authors presented how the MSD behaviors assume different regimes to specific atoms in different places of trehalose aggregate, which suggest that the crossovers emerge from the water-protein interaction. This type of water-biomolecule dynamics was also observed in a number of experimental $^{33-36}$ and simulation ${ }^{26,27,37,38}$ works.

Considering the relevance of the study of water's diffusive behavior, as well as the challenges involved, it is important to understand how much of the slow dynamics is due to the spatial limitations for the water mobility and how much is due to the attraction or repulsion of water to the surface. In the case of the proteins, these two effects seem to be utterly mixed, ${ }^{25-27}$ but we do not know if the combination of water and protein dynamics would be present in other materials. Therefore, in order to test if the water dynamics depends on the nature of the confining surface, we elected to study the hydration water inside the DNA grooves. The water in the grooves is under space constrictions, but the hydrophobicity in principle plays a minor role. ${ }^{39,40}$

Experimental results show that the water at the minor and major groove exhibits a quite distinct thermodynamic and dynamic behavior when compared to bulk water. ${ }^{41-43}$ These studies, however, did not provide the microscopic information necessary to understand how water behaves in these grooves. With the advancement of computational techniques, we can construct structures of nucleic acids, such as hydrated DNA oligonucleotides, which make possible a more detailed study to investigate the properties of water on DNA. ${ }^{43-45}$ In this paper, we analyze how the TIP3P water model behaves inside the DNA minor and major DNA grooves and how the mobility of the confined molecules differs from the behavior in the bulk. We consider the water in the liquid state down to temperatures well below the melting point of liquid-solid, the supercooled water ${ }^{46-48}$ which allow us to understand how the large mobility of bulk water in this region of pressure and low temperatures is affected by the confinement in the grooves.

The manuscript is outlined as follows. In Sec. II, we describe the simulations details, Sec. III presents the analysis of the results including the computation of the radial distribution functions and of the mean square displacements. We show how the water diffusion coefficient depends on the region. In Sec. IV, we present the conclusions.

\section{SIMULATIONS DETAILS}

We performed molecular dynamics (MD) simulations to describe a DNA segment (oligonucleotide) immersed in water. We constructed a B-DNA ${ }^{49}$ structure of the Dickerson-Drew dodecamer [d(CGCGAATTCGCG $)_{2}$ ] using the X3DNA program and converted to AMBER (parmbsc $0^{50}$ ) force field parameters in the GROMACS package. ${ }^{51}$ The dodecamer was solvated using $2.7 \times 10^{4}$ TIP3P water molecules ${ }^{52}$ in a cubic box of side $6.4 \mathrm{~nm}$, with periodic boundary conditions. Since the DNA phosphate groups are negatively charged, 22 sodium cations are added using the program genbox. Since these ions were placed initially in a random distribution, a short molecular dynamics was performed with the $M D$ algorithm in the vacuum, with the use of restraints, allowing the cations to migrate to the vicinity of the phosphates. After this procedure, the solvent molecules were added using the genbox program and a short relaxation simulation was carried out.

The simulation was performed as follows. Initially, the system was equilibrated with a 10 ns MD simulation with only the DNA heavy atoms kept restrained. The production MD simulations were performed in the NpT ensemble using the Berendsen thermostat, ${ }^{53}$ with time constant, $\tau_{T}, 0.5$ ps and the Parrinello-Raman barostat, ${ }^{54}$ with a time constant, $\tau_{p}$ of $2 \mathrm{ps}$, maintaining the pressure at 1 atmosphere. The electrostatic interactions were evaluated with the Particle Mesh Ewald method. ${ }^{55}$ We used an integration step $\Delta t$ of $0.002 \mathrm{ps}$, and the coordinates of all the system's atoms were recorded in a trajectory file every 500 steps.

In order to investigate the structure and dynamics of water located near the DNA grooves, we selected the solvent exposed nitrogen, oxygen, and hydrogen atoms in DNA at the major and minor grooves, and then using these atoms as a reference frame, we followed the dynamics of the water near them. We choose the coupling in the Berendsen thermostat with a time constant of $0.5 \mathrm{ps}$, 250 times the integration step, which is small enough to avoid temperature drift and large enough to exert only a minor effect on the dynamics. Indeed, this idea holds, according to Hünenberger. ${ }^{56}$

The Radial Distribution Function (RDF) $g_{\alpha, \beta}(r)$ between two atoms $\alpha$ and $\beta$ was computed by

$$
g_{\alpha, \beta}(r)=\frac{N_{\alpha}^{-1}}{\left\langle\rho_{\beta}\right\rangle_{\text {local }}} \sum_{i \in \alpha}^{N_{\alpha}} \sum_{j \in \beta}^{N_{\beta}} \frac{\delta\left(r_{i j}-r\right)}{4 \pi r^{2}},
$$

in which $\rho_{\beta}(r)$ is the density of atoms $\beta$ at a distance $r$ of atoms $\alpha$, and $\left\langle\rho_{\beta}\right\rangle_{\text {local }}$ is the average particle density of $\beta$. We employ the water-water and water-DNA radial distribution function at the vicinity of the major and minor grooves to define which water molecules belong to the hydration layer. This distance was used to select the set of water molecules at the vicinity of the grooves. The mobility of these water particles was evaluated by the mean square displacement, namely,

$$
\left\langle(r(t)-r(0))^{2}\right\rangle=\left\langle(\Delta r(t))^{2}\right\rangle,
$$

where $r(0)$ is the position of the particles at the time zero, while $r(t)$ is the position at time $t$. The mobility of the particles was averaged 
over all the water molecules at the vicinity of the grooves. The MSD vs time can be expressed in terms of a power of time as

$$
\left\langle(\Delta r(t))^{2}\right\rangle \propto t^{\mu},
$$

where $\mu=1$ represents a diffusive or Fickian regime, while $\mu<1$ and $\mu>1$ correspond to subdiffusive and superdiffusive regimes, respectively. When the water molecules at the grooves exhibit diffusive regime, we calculated the diffusion coefficient, $D$, from the slope of the plot of the MSD against time as follows:

$$
D=\lim _{t \rightarrow+\infty} \frac{\left\langle(\Delta r(t))^{2}\right\rangle}{2 d t},
$$

where $d$ is the dimension and $\langle\cdots\rangle$ denotes an average over molecules and time origins.

We also computed the number of water-water hydrogen bonds in the vicinity of the grooves. This analysis was performed using geometric criteria: ${ }^{57}$ the existence of a hydrogen bond is considered when the distance between the acceptor and donor atoms is equal to or less than $0.35 \mathrm{~nm}$ and if the hydrogen-donor-acceptor angle is between $0^{\circ}$ and $30^{\circ}$. The number of hydrogen bonds was obtained from the continuous autocorrelation ${ }^{58-60}$ between the molecules $i$ and $j$

$$
C_{H}(t)=\frac{\sum_{i, j} s_{i j}\left(t_{0}\right) s_{i j}\left(t+t_{0}\right)}{\sum_{i, j} s_{i j}\left(t_{0}\right)},
$$

where $s_{i j}$ assumes a unit value when there is a hydrogen bond between the $i$ and $j$ and zero in all other cases.

\section{RESULTS AND ANALYSIS}

First, we explain how we defined the hydration water. Figure 1 presents the radial distribution function of (a) water-water at the bulk and water-DNA at the (b) minor and (c) major grooves. These quantities allow us to estimate the distance defining the hydration layer for each groove at each temperature. The comparison between the first peaks at the radial distribution functions suggests that the distance of $0.3 \mathrm{~nm}$ is a reasonable cutoff for considering water molecules close to the grooves. Similar distances were observed for the hydration layers of proteins. ${ }^{26,27}$

Next, we selected the water near the DNA grooves using the distance of $0.3 \mathrm{~nm}$ as the cutoff distance. The mobility of the water molecules at the DNA grooves was measured for $10^{3} \mathrm{ps}$, which allows us to determine the MSD. Figure 2 compares the MSD of bulk water with the MSD of water at the major and minor grooves for a series of temperatures ranging from 190 to $300 \mathrm{~K}$. At very low temperatures, the water at the grooves exhibits a subdiffusive regime for a short time window, $<10^{2} \mathrm{ps}$, with an exponent $\mu \sim 0.7$ in the behavior of the MSD in Eq. (3). Subdiffusive processes were reported in several systems in which some type of confinement is present, such as the diffusion of the nanoparticles in gels, ${ }^{61}$ lipid membranes, ${ }^{62}$ and others. ${ }^{63-66}$ In this particular case, this represents a transient regime which might be related to the caging effect. For longer times and for higher temperatures, the MSD grows linearly with time with $\mu=1$, a Fickian diffusion in Eq. (3).

Using Eq. (4) applied to the linear, Fickian regime, we determined the diffusion coefficients for water located at different regions
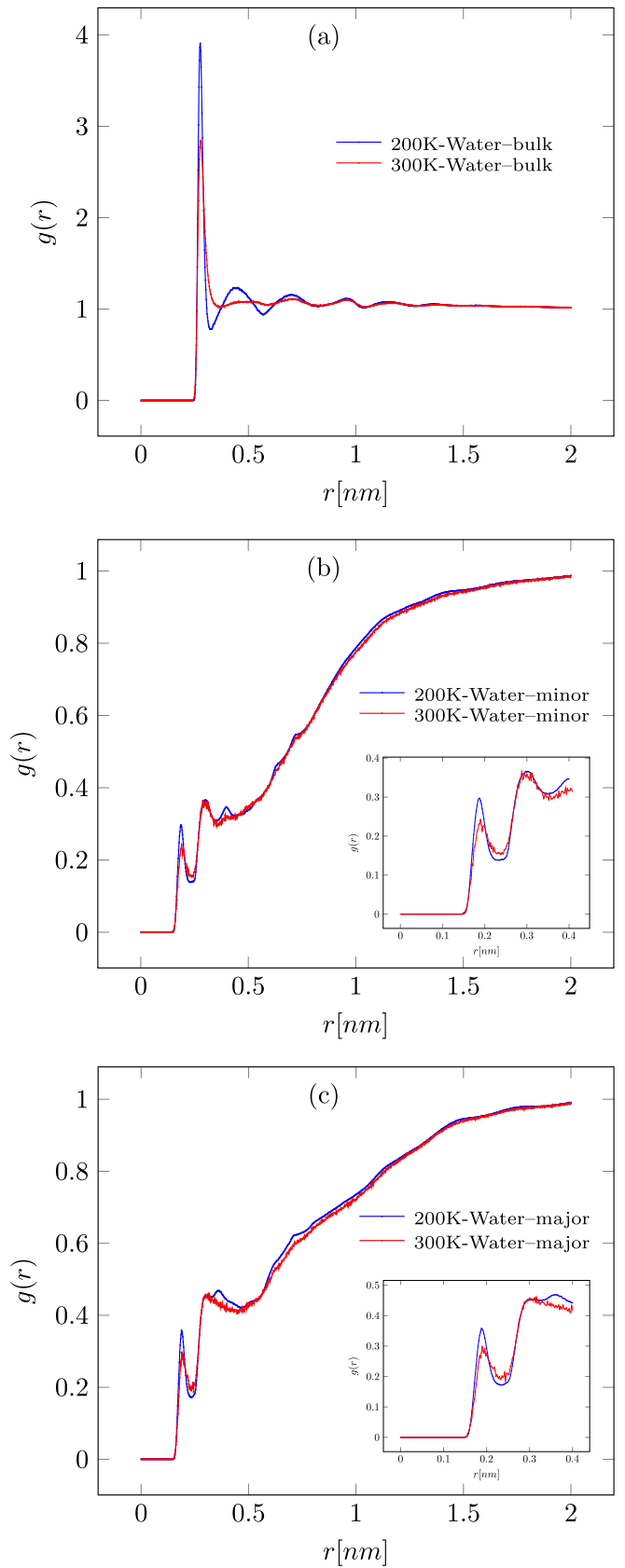

FIG. 1. Radial distribution function for water-water in the (a) bulk, (b) minor, and (c) major grooves at $300 \mathrm{~K}$ (red lines) and $200 \mathrm{~K}$ (blue lines).

of the DNA and for different temperatures ranging from 190 to $300 \mathrm{~K}$. Then, Fig. 3 illustrates the diffusion coefficient vs inverse of temperature $\left(T^{-1}\right)$ for the water in the three scenarios: in bulk, at the major, and at the minor grooves. The diffusion coefficient of TIP3P bulk water in our system refers to the water inside the simulation box containing also the DNA but not interacting with the DNA grooves. Their values are very similar to the diffusion coefficients 

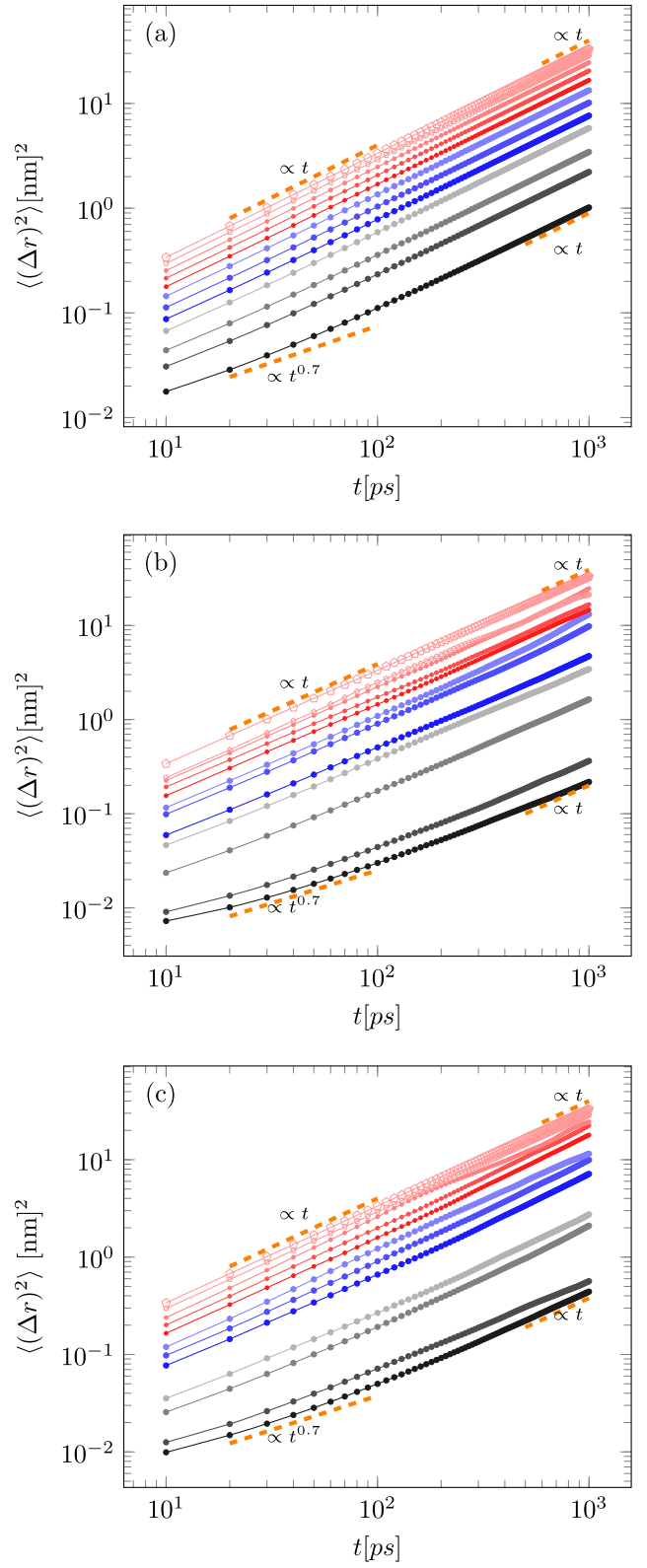

FIG. 2. Mean squared displacement (MSD) of water molecules in the (a) bulk, (b) minor, and (c) major grooves, at different temperatures. Dark black filled circles-190 K, black filled circles-200 K, gray filled circles-210 K, light gray filled circles-220 K, dark blue filled circles-230 K, blue filled circles-240 K, light blue filled circles- $250 \mathrm{~K}$, dark red filled circles-260 K, red filled circles-270 $\mathrm{K}$, light red filled circles-280 K, small open pentagon-290 K, and large open pentagon-300 K

of bulk water in the absence of the DNA, which are higher than the experimental values. ${ }^{67,68}$ The behavior of the logarithm of $D$ vs $1 / T$ is almost linear, what suggests a Arrhenius-type of dynamics. A departure from linearity, however, is observed at very low temperatures. The absence of a bulk water dynamic transition for the TIP3P is not

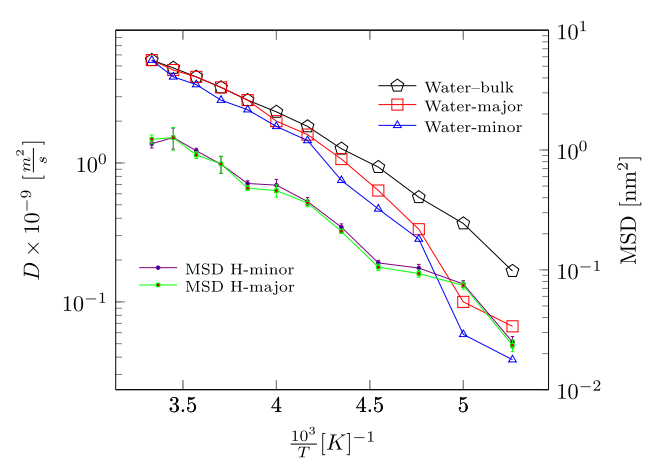

FIG. 3. (left axis) Diffusion coefficient vs the inverse of temperature for water in the bulk (black pentagons), in the major (red squares), and in the minor (blue triangles) grooves. (right axis) MSD of the hydrogen atoms of DNA-grooves at $t=8 \times 10^{2} \mathrm{ps}$. The error bars for the MSDs (green lines and purple lines), estimated by computing 5 separate MSDs in various $10^{3}$ ps blocks of the trajectories.

surprising since the anomalies of water for this model occur at very low temperatures. ${ }^{69}$

For the water in the grooves, Fig. 3 shows that there is a significant breakdown from the linear behavior of $\ln D$ vs $T^{-1}$ not observed in the bulk. ${ }^{68}$ For the major grooves, the departure happens at $T \approx 250 \mathrm{~K}$, while for the water at the minor groove, the breakdown is observed at $T \approx 270 \mathrm{~K}$. This departure from $D \propto e^{-A / T}$ where $A$ is related to the activation energy is consistent with breakdown of Einstein's theory which requires a continuous distribution of particles. In the case of the confined supercooled water, this approximation is unrealistic since there are relevant interactions with the grooves which make the system more structured.

The differences in the dynamics of water molecules in bulk, minor-groove, and major-groove might be due to the geometrical restrictions or to the water-surface interactions. In order to investigate which part of the water dynamics depends on the specific interaction with the surface of the grooves, we analyzed the behavior of the DNA hydrogen by means of the MSD. Figure 3 shows the MSD of the hydrogen at the DNA minor and major grooves at $t=8 \times 10^{2} \mathrm{ps}$. The data show no dynamic transition but three regions of a nonlinear behavior. The comparison between the MSD of the DNA hydrogen and the diffusion of water confined in the grooves seems to show no strong correlation; however, at low temperatures, both the MSD and the diffusion are very low. Our results are consistent with similar observations of the relation between the $\mathrm{H}$-bond lifetimes and the residence times of oxygen atoms of water in the first solvation shell which are slower at the minor grooves when compared to other regions. ${ }^{7}$

In order to check how strong is the interaction of water in the grooves at low temperatures, when the water dynamics becomes slow and violates the Stokes-Einstein relation, we computed the water-water hydrogen bond correlation functions. Figure 4 shows the autocorrelation function (ACF) of $\mathrm{H}$-bonds vs time, for water in the bulk and in the vicinity of the minor and major grooves for all studied temperatures. Figure 4 indicates that the water molecules at the DNA grooves form $\mathrm{H}$-bonds that are more stable than those in the bulk. A similar result was also obtained by analyzing water on the surface of the antifreeze protein. ${ }^{73}$ As the temperature increases, 

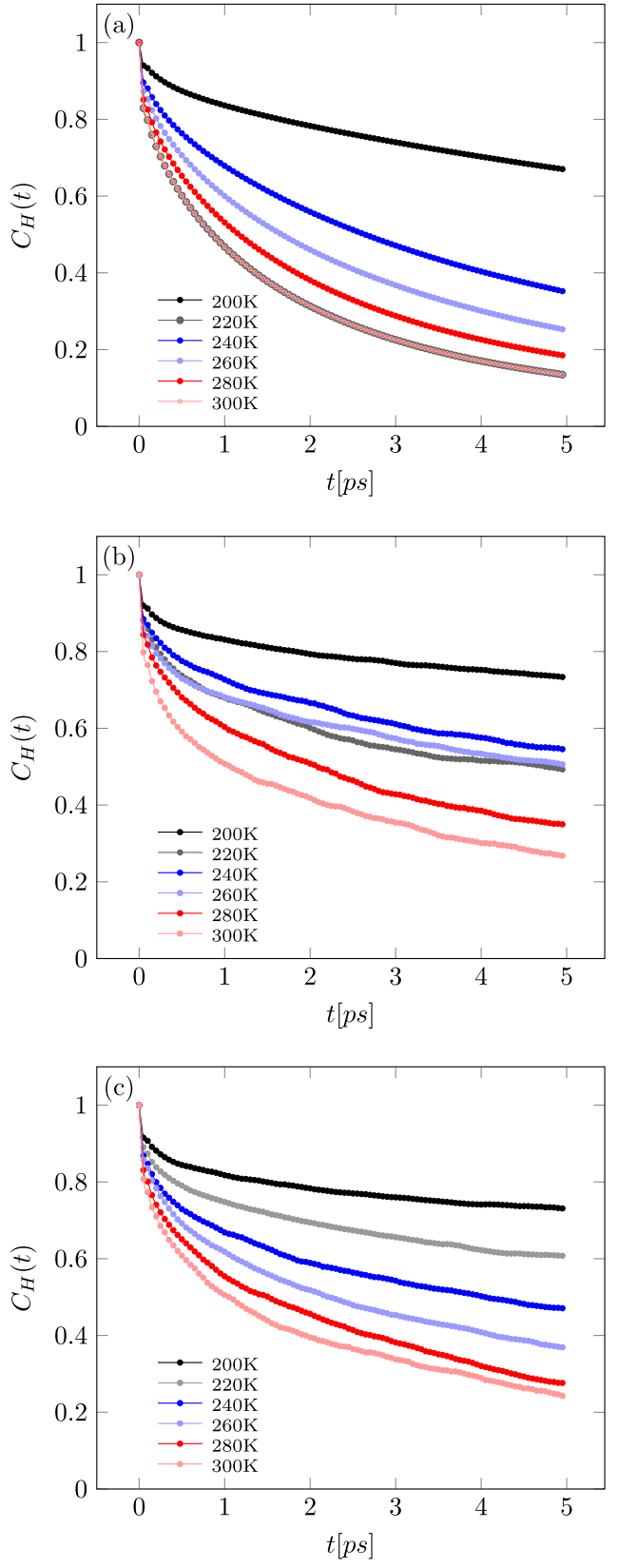

FIG. 4. Autocorrelation functions of water-water $\mathrm{H}$-bonds $C_{H}(t)$ in the (a) bulk, (b) minor, and (c) major grooves for selected temperatures.

the lifetime of the $\mathrm{H}$-bonds of water at the major grooves becomes similar to the lifetime of the H-bonds of water in the bulk, but the $\mathrm{H}$-bonds of water at the minor grooves exhibit higher stability and lower diffusion. Our results indicate that the mechanism behind the slow dynamics is associated with stronger $\mathrm{H}$-bonds between supercooled water in grooves in relation with supercooled water in bulk.

\section{CONCLUSIONS}

In this work, we investigated the differences in the TIP3P water dynamics in three regions of the DNA-water solution: at the major and minor grooves and in the bulk. Considering only the water molecules that do not leave the DNA grooves, we show that at low temperatures, the molecules in the minor and major grooves move more slowly relative to bulk water. In addition, we observed that for the diffusion coefficient for a bulk system, an Arrhenius behavior is observed. However, for the water in the major groove, the departure from the linear behavior $\ln D \propto 1 / T$ is observed for $T<250 \mathrm{~K}$, while for the water in the minor groove, the breakdown is seen at $T<270 \mathrm{~K}$.

In order to understand the mechanisms behind the anomalous behavior of supercooled water, we calculated the autocorrelation function of the $\mathrm{H}$-bonds and we showed that the molecules interact with a strong correlation with the minor and major grooves of the DNA at low temperatures, which justifies the different behaviors for water dynamics in the bulk and in the DNA grooves.

\section{ACKNOWLEDGMENTS}

We thank the Brazilian agencies CNPq, INCT-FCx, and Capes for the financial support.

\section{REFERENCES}

${ }^{1}$ M. Chaplin, “Seventh-two anomalies of water” (2014); available at http://www1. lsbu.ac.uk/water/anmlies.html, 2014

${ }^{2}$ P. A. Netz, F. W. Starr, H. E. Stanley, and M. C. Barbosa, J. Chem. Phys. 115, 344 (2001).

${ }^{3}$ U. Raviv, P. Laurat, and J. Klein, Nature 413, 51 (2001).

${ }^{4}$ P. Ternes, E. Salcedo, and M. C. Barbosa, Phys. Rev. E 97, 033104 (2018).

${ }^{5}$ M. H. Köhler, J. R. Bordin, and M. C. Barbosa, J. Chem. Phys. 148, 222804 (2018).

${ }^{6}$ P. Gallo, M. Rovere, and S.-H. Chen, J. Phys.: Condens. Matter 22, 284102 (2010).

${ }^{7}$ M. H. Köhler, J. R. Bordin, C. F. de Matos, and M. C. Barbosa, Chem. Eng. Sci. 203, 54 (2019).

${ }^{8}$ M. H. Köhler, J. R. Bordin, L. B. da Silva, and M. C. Barbosa, Phys. Chem. Chem. Phys. 19, 12921 (2017).

${ }^{9}$ S. P. Surwade, S. N. Smirnov, I. V. Vlassiouk, R. R. Unocic, G. M. Veith, S. Dai, and S. M. Mahurin, Nat. Nanotechnol. 10, 459 (2015).

${ }^{10}$ J. R. Werber, C. O. Osuji, and M. Elimelech, Nat. Rev. Mater. 1, 16018 (2016).

${ }^{11}$ L. Zhou, Y. Tan, J. Wang, W. Xu, Y. Yuan, W. Cai, S. Zhu, and J. Zhu, Nat. Photonics 10, 393 (2016).

${ }^{12}$ Y. Levy and J. N. Onuchic, Proc. Natl. Acad. Sci. U. S. A. 101, 3325 (2004).

${ }^{13}$ Y. Levy and J. N. Onuchic, Annu. Rev. Biophys. Biomol. Struct. 35, 389 (2006).

${ }^{14}$ S. Kalia and L. Avérous, Biopolymers: Biomedical and Environmental Applications (John Wiley \& Sons, 2011), Vol. 70.

${ }^{15}$ B. C. Steele and A. Heinzel, Materials for Sustainable Energy: A Collection of Peer-Reviewed Research and Review Articles from Nature Publishing Group (World Scientific, 2011), pp. 224-231.

${ }^{16}$ K. Takata, T. Matsuzaki, and Y. Tajika, Prog. Histochem. Cytochem. 39, 1 (2004).

${ }^{17}$ T. Zeuthen, J. Membr. Biol. 234, 57 (2010).

${ }^{18}$ E. Yamamoto, T. Akimoto, M. Yasui, and K. Yasuoka, Sci. Rep. 4, 4720 (2014).

${ }^{19}$ P. Nelson, Biological Physics (W. H. Freeman, New York, 2004).

${ }^{20}$ S. K. Pal and A. H. Zewail, Chem. Rev. 104, 2099 (2004).

${ }^{21}$ S. Shin and A. P. Willard, J. Chem. Theory Comput. 14, 461 (2018).

${ }^{22}$ A. J. Patel, P. Varilly, and D. Chandler, J. Phys. Chem. B 114, 1632 (2010).

${ }^{23}$ M. Mehlhorn, S. Schnur, A. Groß, and K. Morgenstern, ChemElectroChem 1, 431 (2014). 
${ }^{24}$ S.-H. Chong and S. Ham, Sci. Rep. 7, 8744 (2017).

${ }^{25}$ A. Iorio, G. Camisasca, and P. Gallo, J. Mol. Liq. 282, 617 (2019).

${ }^{26}$ M. H. Köhler, R. C. Barbosa, L. B. Silva, and M. C. Barbosa, Physica A 468, 733 (2017).

${ }^{27}$ R. C. Barbosa and M. C. Barbosa, Physica A 439, 48 (2015).

${ }^{28}$ P. Tan, Y. Liang, Q. Xu, E. Mamontov, J. Li, X. Xing, and L. Hong, Phys. Rev. Lett. 120, 248101 (2018).

${ }^{29}$ R. Metzler, Physics 11, 59 (2018)

${ }^{30}$ R. Metzler and J. Klafter, Phys. Rep. 339, 1 (2000).

${ }^{31}$ T. E. de Oliveira, C. M. Marques, and P. A. Netz, Phys. Chem. Chem. Phys. 20, 10100 (2018).

${ }^{32}$ S.-H. Chen, L. Liu, E. Fratini, P. Baglioni, A. Faraone, and E. Mamontov, Proc. Natl. Acad. Sci. U. S. A. 103, 9012 (2006).

${ }^{33}$ G. Schirò, M. Fomina, and A. Cupane, J. Chem. Phys. 139, 121102 (2013).

${ }^{34}$ F. Mallamace, C. Corsaro, D. Mallamace, S. Vasi, C. Vasi, and H. E. Stanley, J. Chem. Phys. 141, 18C504 (2014).

${ }^{35}$ F. Mallamace, C. Corsaro, D. Mallamace, P. Baglioni, H. E. Stanley, and S.-H. Chen, J. Phys. Chem. B 115, 14280 (2011).

${ }^{36}$ M.-C. Bellissent-Funel, A. Hassanali, M. Havenith, R. Henchman, P. Pohl, F. Sterpone, D. van der Spoel, Y. Xu, and A. E. Garcia, Chem. Rev. 116, 7673 (2016).

${ }^{37}$ P. Kumar, Z. Yan, L. Xu, M. G. Mazza, S. V. Buldyrev, S.-H. Chen, S. Sastry, and H. E. Stanley, Phys. Rev. Lett. 97, 177802 (2006).

${ }^{38}$ A. K. Pathak and T. Bandyopadhyay, J. Chem. Theory Comput. 15, 2706 (2019).

${ }^{39}$ B. Schneider, K. Patel, and H. M. Berman, Biophys. J. 75, 2422 (1998).

${ }^{40}$ R. Rohs, S. M. West, A. Sosinsky, P. Liu, R. S. Mann, and B. Honig, Nature 461, 1248 (2009).

${ }^{41}$ S. K. Pal, L. Zhao, and A. H. Zewail, Proc. Natl. Acad. Sci. U. S. A. 100, 8113 (2003).

${ }^{42}$ L. A. Kuhn, M. A. Siani, M. E. Pique, C. L. Fisher, E. D. Getzoff, and J. A. Tainer, J. Mol. Biol. 228, 13 (1992).

${ }^{43}$ B. Jana, S. Pal, P. K. Maiti, S.-T. Lin, J. T. Hynes, and B. Bagchi, J. Phys. Chem. B 110, 19611 (2006).

${ }^{44}$ P. L. Privalov, A. I. Dragan, C. Crane-Robinson, K. J. Breslauer, D. P. Remeta, and C. A. Minetti, J. Mol. Biol. 365, 1 (2007).

${ }^{45}$ C. G. Ricci, A. S. de Andrade, M. Mottin, and P. A. Netz, J. Phys. Chem. B 114, $9882(2010)$

${ }^{46}$ P. Netz, F. Starr, M. C. Barbosa, and H. Stanley, Physica A 314, 470 (2002), horizons in complex systems.

${ }^{47}$ P. Gallo and H. E. Stanley, Science 358, 1543 (2017).
${ }^{48}$ C. Goy, M. A. C. Potenza, S. Dedera, M. Tomut, E. Guillerm, A. Kalinin, K.-O. Voss, A. Schottelius, N. Petridis, A. Prosvetov, G. Tejeda, J. M. Fernández, C. Trautmann, F. Caupin, U. Glasmacher, and R. E. Grisenti, Phys. Rev. Lett. 120, 015501 (2018).

${ }^{49}$ R. R. Sinden, DNA Structure and Function (Elsevier, 2012).

${ }^{50}$ A. Pérez, I. Marchán, D. Svozil, J. Sponer, T. E. Cheatham, C. A. Laughton, and M. Orozco, Biophys. J. 92, 3817 (2007).

${ }^{51}$ B. Hess, C. Kutzner, D. Van Der Spoel, and E. Lindahl, J. Chem. Theory Comput. 4, 435 (2008).

${ }^{52}$ W. L. Jorgensen, J. Chandrasekhar, J. D. Madura, R. W. Impey, and M. L. Klein, J. Chem. Phys. 79, 926 (1983).

${ }^{53}$ H. J. C. Berendsen, J. P. M. Postma, W. F. van Gunsteren, A. DiNola, and J. Haak, J. Chem. Phys. 81, 3684 (1984).

${ }^{54}$ M. Parrinello and A. Rahman, J. Appl. Phys. 52, 7182 (1981).

${ }^{55}$ T. I. Cheatham, J. Miller, T. Fox, T. Darden, and P. Kollman, J. Am. Chem. Soc. 117, 4193 (1995).

${ }^{56}$ P. H. Hünenberger, Adv. Polym. Sci. 173, 105 (2005).

${ }^{57}$ S. Pronk, S. Páll, R. Schulz, P. Larsson, P. Bjelkmar, R. Apostolov, M. R. Shirts, J. C. Smith, P. M. Kasson, D. Van Der Spoel et al., Bioinformatics 29, 845 (2013).

${ }^{58}$ D. C. Rapaport, Mol. Phys. 50, 1151 (1983).

${ }^{59}$ A. Luzar, J. Chem. Phys. 113, 10663 (2000).

${ }^{60}$ D. van der Spoel, P. J. van Maaren, P. Larsson, and N. Tîmneanu, J. Phys. Chem. B 110, 4393 (2006).

${ }^{61}$ P. A. Netz and T. Dorfmüller, J. Chem. Phys. 103, 9074 (1995).

${ }^{62}$ R. Metzler, J.-H. Jeon, and A. G. Cherstvy, Biochim. Biophys. Acta, Biomembr. 1858, 2451 (2016).

${ }^{63}$ E. Barkai, Chem. Phys. 284, 13 (2002).

${ }^{64}$ M. A. F. dos Santos, Fractal Fractional 2, 20 (2018).

${ }^{65}$ M. A. F. dos Santos and I. S. Gomez, J. Stat. Mech.: Theory Exp. 2018, 123205.

${ }^{66}$ M. A. F. dos Santos, J. Stat. Mech.: Theory Exp. 2019, 033214.

${ }^{67}$ L. J. Kinnaman, R. M. Roller, and C. S. Miller, J. Chem. Educ. 95, 888 (2018).

${ }^{68}$ E. E. S. Ong and J.-L. Liow, Fluid Phase Equilib. 481, 55 (2019).

${ }^{69}$ W. L. Jorgensen and C. Jenson, J. Comput. Chem. 19, 1179 (1998).

${ }^{70}$ J. Zavadlav, J. R. Podgornik, and M. Praprotnik, J. Chem. Theory Comput. 11, 5035 (2015).

${ }^{71}$ J. Zavadlav, R. Podgornik, M. N. Melo, S. J. Marrink, and M. Praprotnik, Eur. Phys. J.: Spec. Top. 225, 1595 (2016).

${ }^{72}$ J. Zavadlav, J. R. Podgornik, and M. Praprotnik, Sci. Rep. 7, 4775 (2017).

${ }^{73}$ Y. Xu, R. Gnanasekaran, and D. M. Leitner, J. At., Mol., Opt. Phys. 2012, 1. 